Case Report

\title{
A Delicate Surgical Resection to Treat a Proximal Pulmonary Adenoid Cystic Carcinoma
}

\author{
N. Belloumi ${ }^{1},{ }^{1,2}$ H. Baili, ${ }^{1}$ M. Abdennadher, ${ }^{3}$ C. Habbouria, ${ }^{1}$ I. Bachouch, ${ }^{1}$ I. Bouassida, ${ }^{3}$ \\ S. Zairi, ${ }^{3}$ F. Chermiti Ben Abdallah, ${ }^{1,2}$ S. Fenniche, ${ }^{1,2}$ H. Zribi, ${ }^{3}$ and A. Marghli ${ }^{3}$ \\ ${ }^{1}$ Pulmonology Department Pavilion 4, Abderrahmen Mami Hospital, Tunisia \\ ${ }^{2}$ Faculty of Medicine of Tunis, University of Tunis El Manar, Tunisia \\ ${ }^{3}$ Thoracic Surgery Department, Abderrahmen Mami Hospital, Tunisia
}

Correspondence should be addressed to N. Belloumi; nidhalbelloumi@gmail.com

Received 7 February 2021; Accepted 12 June 2021; Published 3 July 2021

Academic Editor: Alessandro Bandiera

Copyright (c) 2021 N. Belloumi et al. This is an open access article distributed under the Creative Commons Attribution License, which permits unrestricted use, distribution, and reproduction in any medium, provided the original work is properly cited.

\begin{abstract}
Pulmonary adenoid cystic carcinoma (PACC) is an exceedingly rare tumor of low-grade malignancy. Diagnosis is often late, so the tumoral process may be huge at presentation. Surgical resection could be challenging, and the recurrence rate would be greater. We report, hereby, a case of proximal PACC with involvement of the carina in a young male adult, without respiratory distress. Surgical resection was performed through a left pneumonectomy followed by a complex trachea-bronchoplastic procedure. During the operative time, the assisted ventilatory mode was carefully chosen. No adjuvant treatment was needed. Our patient is still under clinicoradiological surveillance and remains disease-free.
\end{abstract}

\section{Introduction}

Pulmonary adenoid cystic carcinoma (PACC) is an exceedingly rare tumor of low-grade malignancy, accounting for 0.09 to $0.2 \%$ of all lung cancers [1]. Diagnosis is often late because the symptoms appear only after the tumor infiltrates the proximal airways. Symptoms are usually noncharacteristic, starting with cough and leading to dyspnea on exertion [2]. PACC has an unpredictable growth behavior. We report a case for broadening the sight of this disease, requesting a complex surgical procedure.

\section{Case Report}

A 29-year-old male patient with no past medical history was admitted to our department to explore a right basal thoracic pain mentioned two months ago aggravated by an acute onset dyspnea. He was an active smoker and worked as a baker. On examination, the patient was afebrile and eupneic, no oxygen was needed, and auscultation found a decrease in vesicular murmur on the left with dullness on percussion.
Chest X-ray revealed a homogeneous pulmonary opacity occupying the entire left pulmonary field with a mediastinum lifted to the left. Bronchial endoscopy revealed a hypervascularized bud obstructing the entrance to the left mainstem bronchus (Figure 1). The anatomopathological exam of bronchial biopsies showed a bronchial mucosa infiltrated by a carcinomatous proliferation arranged in tubular structures, cribriform masses, small lobules, and cords. Tumor cells were monomorphic, cuboidal, or basophilic with sparse cytoplasm and little atypical dense chromatin ovoid nuclei. Mitoses were rare. The stroma was fibrohyaline with myxoïd islands and several layers of mucoid material. This histological appearance was very suggestive of the diagnosis of adenoid cystic carcinoma. CT scan had shown a mediastinal gangliotumoral complex of $52 \times 38 \mathrm{~mm}$ obstructing the left main bronchus with an ipsilateral pulmonary collapse. The process invaded the carina and the lower trachea (Figure 2). No distant metastasis was found.

The patient underwent a left pneumonectomy with a section of the left mainstem bronchus and lymph node dissection through a left thoracotomy. Then, a right thoracotomy 

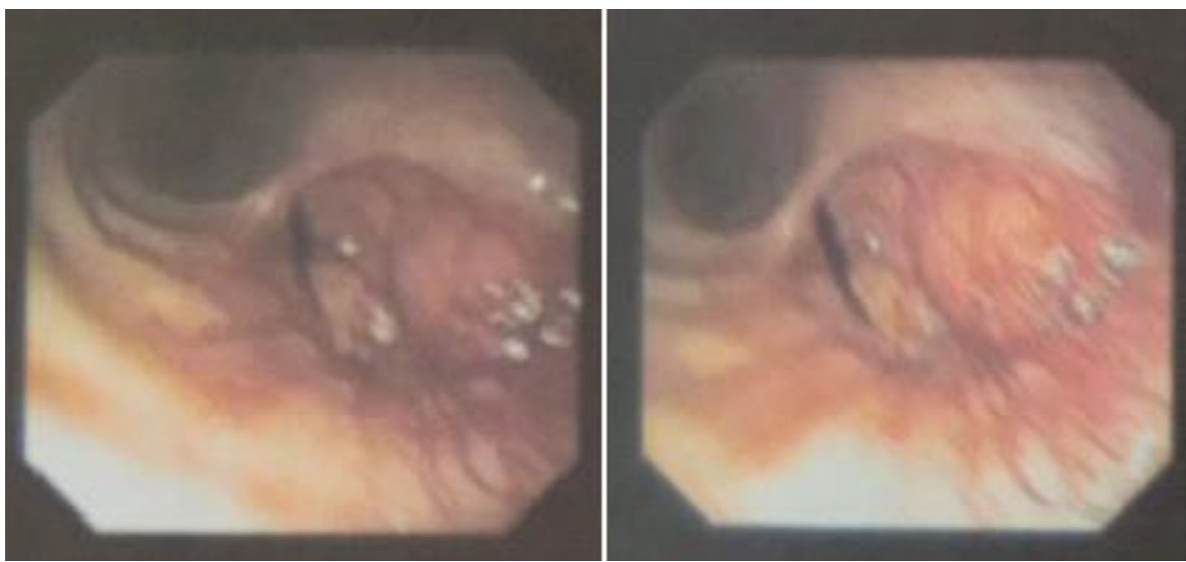

FIGURE 1: Bronchial endoscopy revealed a hypervascularized bud obstructing the left mainstem bronchus.

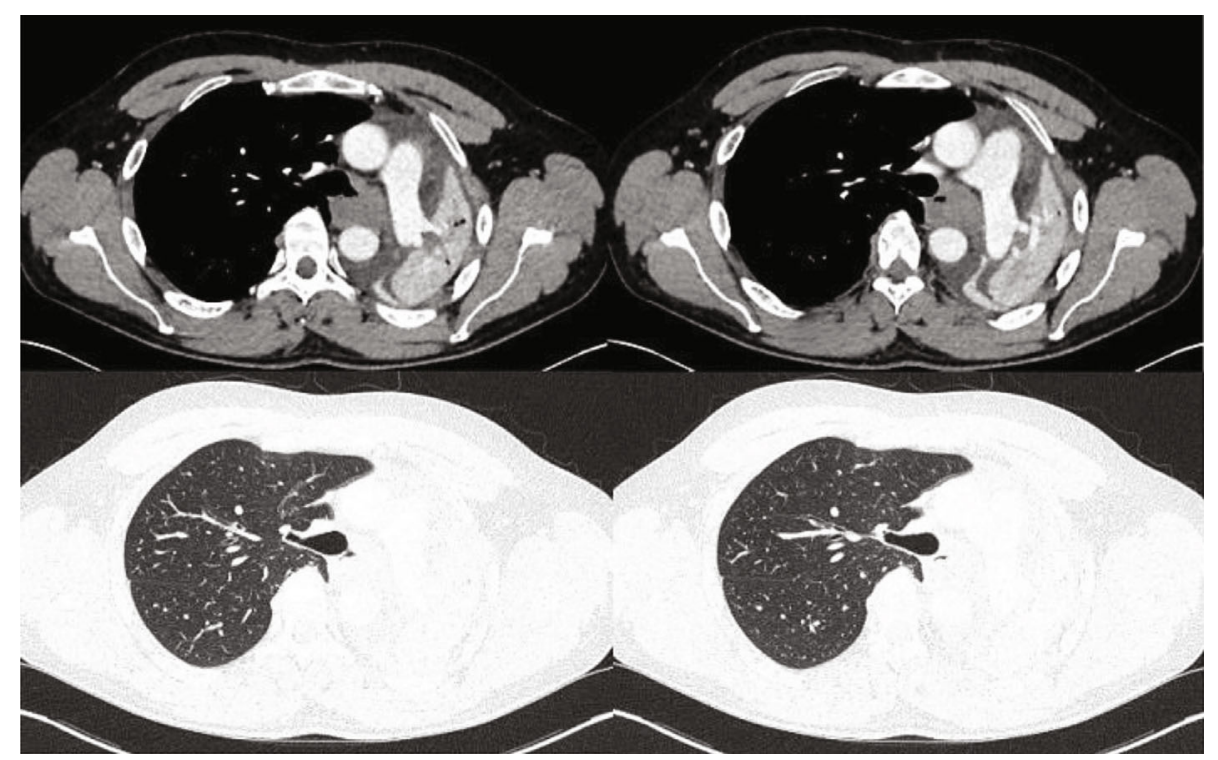

FIGURE 2: CT scan findings before the surgical procedure: a mediastinal gangliotumoral complex obstructing the left main bronchus, invasion of the carina and the lower trachea with an ipsilateral pulmonary collapse.

was performed. Selective high-frequency jet ventilation was performed through the right main bronchus. The tracheabronchial section was enlarged to the lower part $(4 \mathrm{~cm})$ of the trachea and the carina. A terminoterminal anastomosis restored continuity of the distal part of the trachea and the right mainstem bronchus. Anastomotic sutures using 4-0 polydioxanone suturing thread were placed around the entire circumference, then ligated making ends meet.

Postoperatively, the patient was given oxygen therapy at home for a month. The CT scan performed 9 months after surgery showed no signs of regional or distant recurrence. Endoscopic control objectified a barely visible bulge at the right strain bronchus that marks trachea-bronchial plasty without narrowing of the lumen. A clinicoradiological follow-up is ensured for 24 months nowadays (Figure 3). The patient regained his professional career.

\section{Discussion}

PACC is uncommon but represents the predominant type of salivary gland-type lung carcinomas [3]. So far, several large studies and more case reports have been reported in the literature about PACC. This tumor is unrelated to smoking, it appears usually in the fifth decade, and there is no particular sex predilection $[2,4,5]$. Our patient was, so, younger than usually reported.

ACC develops mostly in the trachea, the mainstem, or the lobar bronchi $[6,7]$. The tumor is presumed to derive from a primitive tracheobronchial gland cell. PACC is usually solitary, nodular, or polypoid masses that protrude the cartilage and infiltrate the peribronchial tissue $[6,7]$.

Histologically, ACC shows differentiation towards ductal and myoepithelial cells [6]. Authors already described 


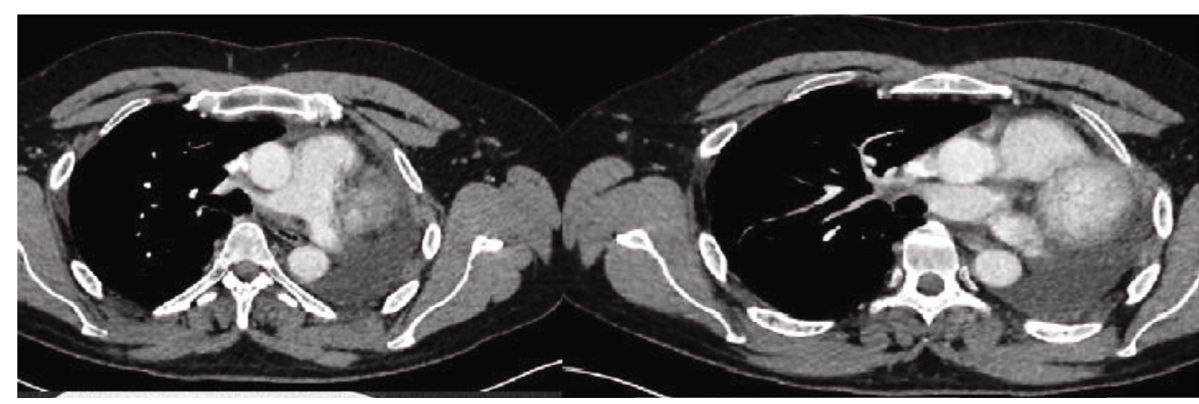

Figure 3: CT scan after left pneumonectomy.

cribriform, tubular, or solid patterns. The most important and classical feature is the "cribriform" pattern where nests of tumor cells have a soft yellow-white cut surface. Most ACC show a pattern mixture. Immunostaining is not necessary for the diagnosis but can serve out in several special cases notably in solid or tubular ACCs [2]. Tumor cells express smooth muscle actin and myosin, the protein S100, and CD117 [8]. Overexpression of p53 and Ki-67 with loss of myoepithelial markers was found in high-grade tumors.

ACC tends to submucosal extension linked to the arrangement of the accessory salivary glands, located between the cartilage and the tracheal fibromuscular membrane, at the level of the posterior wall of the trachea. It shows up in CT scan as an intraluminal mass extending through the tracheal wall. Sometimes, the lesion may present as a circumferential parietal thickening $[2,9]$. Respiratory functional assessment is mandatory after the diagnosis of ACC. The fixed airway obstruction with decreased inspiratory and peak expiratory flow is due to the intraluminal narrowing [1].

Treatment options for advanced PACC patients are limited. Currently, the best treatment relies on complete resection with tracheobronchial plasty depending on tumoral location and extension. Developed surgical and anesthesiological procedures would make even wide tumors resectable. Contraindications to surgery include mediastinal lymph nodes, vascular involvement, tracheal invasion for more than $50 \%$ of its length, and distant metastases [10].

The extent of surgical resection and adjuvant radiotherapy may influence the prognosis of ACC. In a 30-year study of ACC of the airway, Calzada et al. found a high progressionfree survival rate for patients who underwent surgical resection followed by adjuvant radiotherapy [11]. Early radical resection and radiotherapy are associated with a low risk of local recurrence of PACC.

\section{Conclusions}

Lung adenoid cystic carcinoma is a rare lung cancer with protracted but unpredictable growth behavior. Diagnosis and treatment are not yet fully consensual. For huge resectable masses, postoperative radiation would cure the microscopically residual tumor of the mediastinum. The mainstay of treatment is based upon combined surgical resection to radiotherapy. A long-term follow-up is required due to the risk of local or distant recurrence.

\section{Data Availability}

Data are available upon request to the corresponding author, email=nidhalbelloumi@gmail.com.

\section{Conflicts of Interest}

There are no interests related to the manuscript theme.

\section{References}

[1] R. Kundu, U. Handa, R. S. Punia, A. Dass, and V. Saini, "Adenoid cystic carcinoma: a study of 19 cases of salivary and extrasalivary tumours diagnosed by fine needle aspiration cytology," Diagnostic cytopathology, vol. 46, no. 12, pp. 10041009, 2018

[2] H. M. Desai, R. Thakare, G. P. Amonkar, V. Karkhanis, and J. M. Joshi, "Adenoid cystic carcinoma of the trachea," Indian Journal of Pathology and Microbiology, vol. 58, no. 4, pp. 516518, 2015.

[3] Z. Huo, H. Wu, S. Li, and Z. Liang, "Molecular genetic studies on EGFR, KRAS, BRAF, ALK, PIK3CA, PDGFRA, and DDR2 in primary pulmonary adenoid cystic carcinoma," Diagnostic pathology, vol. 15, 2015.

[4] F. Zhu, Z. Liu, Y. Hou et al., "Primary salivary gland-type lung cancer: clinicopathological analysis of 88 cases from China," Journal of Thoracic Oncology, vol. 8, no. 12, pp. 1578-1584, 2013.

[5] D. Debieuvre, C. Locher, A.-C. Neidhardt et al., "Ten-year evolution in non-small-cell lung cancer according to sex. Results of the KBP-2010-CPHG study by the College of General Hospital Respiratory Physicians," Revue des maladies respiratoires, vol. 31, no. 9, pp. 805-816, 2014.

[6] O. Kawashima, T. Hirai, M. Kamiyoshihara, S. Ishikawa, and Y. Morishita, "Primary adenoid cystic carcinoma in the lung: report of two cases and therapeutic considerations," Lung Cancer, vol. 19, no. 3, pp. 211-217, 1998.

[7] M.-M. Hu, Y. Hu, J.-B. He, and B.-L. Li, "Primary adenoid cystic carcinoma of the lung: clinicopathological features, treatment and results," Oncology letters, vol. 9, no. 3, pp. 14751481, 2015.

[8] J. Jaso and R. Malhotra, "Adenoid cystic carcinoma," Archives of pathology \& laboratory medicine, vol. 135, no. 4, pp. 511515, 2011.

[9] R. Maroldi, D. Farina, A. Borghesi, A. Marconi, and E. Gatti, "Perineural tumor spread," Neuroimaging clinics of North America, vol. 18, no. 2, pp. 413-429, 2008, xi. 
[10] M. Li, B.-R. Zhao, S.-Q. Liu et al., "Mutational landscape and clonal diversity of pulmonary adenoid cystic carcinoma," Cancer Biology \& Therapy, vol. 19, no. 10, pp. 898-903, 2018.

[11] A. P. Calzada, M. Miller, C. K. Lai, D. A. Elashoff, E. Abemayor, and M. A. St John, "Adenoid cystic carcinoma of the airway: a 30-year review at one institution," American journal of otolaryngology, vol. 33, no. 2, pp. 226-231, 2012. 